\title{
Effect of Health Qigong New Skills on Serum SOD and MDA of Quinquagenarian
}

\author{
Chuikun $\mathrm{Li}^{\mathrm{a}}$, Yeting Zhang ${ }^{\mathrm{b}}$ \\ College of Physical Education, Chengdu University, Chengdu 610106, China. \\ a23522443@qq.com, b690785469@qq.com
}

Keywords: Health Qigong, serum, SOD, MDA.

\begin{abstract}
Objective: To observe the health Qigong new skills on the change of radical superoxide dismutase (SOD) and malondialdehyde (MDA) of quinquagenarian. Methods: In this paper, quinquagenarian who were basically healthy but don't toe part in physical exercise in Chengdu, Sichuan Province object. The people divided into four groups according to gender and age, each group of 12 people. Before practice, took every group's blood to tested serum SOD and MDA. Under the guidance of the instructor, the subjects practiced five sets of new health Qigong. Practice at least once a day for each set of skills. After 8 weeks practiced, took every group's blood to tested serum the activity of SOD was measured by the xanthine oxidase method and the MDA content in the thiobarbitic acid (TBA) reaction colorimetric method. Results: 1. The activity of serum SOD: the serum SOD activity after exercise in middle men's group and middle women's group was higher than that before exercise, but there was no significant difference $(\mathrm{P}=0.332, \mathrm{P}=0.218)$. The serum SOD activity in older men's group and middle-aged women's group after exercise was significantly higher than before exercise, and there was a significant difference $(\mathrm{P}=0.001, \mathrm{P}=0.003) .2$. The content of MDA: the serum MDA activity of middle men's group, older men's group and middle women's group were decreased and there was no significant difference compared with that before exercise $(\mathrm{P}=0.088$, $\mathrm{P}=0.118, \mathrm{P}=0.145)$. There was a significant difference between the serum MDA content and before exercise in the elderly women group $(\mathrm{P}=0.024)$. Conclusion: After 8 weeks the health Qigong new skills, which can improve the serum SOD activity and reduce MDA content in serum and reduce the degree of lipid peroxidation. Prompt accurate grasp of the action essentials and a long and serious exercise in the health Qigong new skills is good for the prevention of cardiovascular disease in the elderly and the process of slowing down the aging process.
\end{abstract}

\section{Introduction}

Health Qigong•new skills is a popular national traditional sports and fitness method which is popular among the masses in ancient China, which combines physical activity, breathing and breathing, and the combination of psychological adjustment and national traditional sports and fitness. The state general administration of sport's fitness qigong management center has compiled and created five new skills: Health Qigong•Daoyin Yangsheng Gong 12 Methods and Health Qigong•Shi Er Duan Jin(Beijing Sport University), Health Qigong•Tai Ji Yang Sheng Zhang(Tsinghua University), Health Qigong•Ma Wang Dui Dao Yin Shu(Shanghai University of Sport) and Health Qigong•Da Wu(Wuhan Sports University)[1].

In 1956, Denham Harman proposed the free radical theory of aging mechanism. With the increase of age, the body's antioxidant defense system function decreases, and free radical generation increases, and the removal ability decreases, causing the dynamic balance of physiological function to be destroyed[2].

New achievement method practitioners as the research object, this study is to observe the new achievement method practitioner before and after serum superoxide dismutase (SOD) and lipid peroxide degradation products malondialdehyde (MDA) of metabolism. This paper aims to explore the influence mechanism of the new method on the aging process. 


\section{Study Subjects and Methods}

\subsection{Study Subjects.}

In this paper, 48 people with basic health but not frequent physical exercise in Chengdu, Sichuan province were studied. The subjects will practice the time of their work by the time of 7:00 to 9:00 in the morning. According to the gender and age ,they are divided into the following four groups: the age of middle men's group is $56.8 \pm 2.31$, the age of old men's group is $67.7 \pm 2.14$, the age of middle women's group is $57.4 \pm 2.47$, the age of elderly women's group is $66.5 \pm 2.28$, and each group of 12 people[3].

\subsection{The Research Methods.}

Under the guidance of the instructor, the subjects practiced the following five sets of new skills such as: Health Qigong•Daoyin Yangsheng Gong 12 Methods, Health Qigong•Shi Er Duan Jin, Health Qigong•Tai Ji Yang Sheng Zhang, Health Qigong•Ma Wang Dui Dao Yin Shu and Health Qigong $\bullet$ Da Wu. Practice at least once a day for each set of skills. The subjects performed at least 1 hour of exercise each day for 8 weeks[3]. The activity of SOD was measured by the xanthine oxidase method and the MDA content in the thiobarbitic acid (TBA) reaction colorimetric method. The kit was strictly operated according to the Nanjing Jiancheng Bioengineering Institute.

\section{Statistical Methods}

SPSS 18.0 statistical software was used to analyze the data, and the results of the experiment were expressed as the mean \pm standard deviation $\left(\bar{X}_{ \pm \mathrm{S}}\right)$, and the variance analysis was used between the groups.

\section{The Experimental Results}

\subsection{The Effect of the New Method on Free Radical SOD in Each Group.}

According to table 1, the serum SOD activity after exercise in middle men's group and middle women's group was higher than that before exercise, but there was no significant difference $(\mathrm{P}=0.332$, $\mathrm{P}=0.218$ ). The serum SOD activity in older men's group and middle women's group after exercise was significantly higher than before exercise, and there was a significant difference $(\mathrm{P}=0.001, \mathrm{P}=0.003)$.

\subsection{The Effect of the Health Qigong New Skills on Free Radical MDA in Each Group.}

According to table 1, the serum MDA activity of middle men's group, older men's group and middle women's group were decreased and there was no significant difference compared with that before exercise $(\mathrm{P}=0.088, \mathrm{P}=0.118, \mathrm{P}=0.145)$. There was a significant difference between the serum MDA content and before exercise in the elderly women group $(\mathrm{P}=0.024)$.

\begin{tabular}{ccccccc}
\multicolumn{5}{c}{ Table 1. Changes of SOD and MDA before and after exercise in each group $\left(\bar{X}_{ \pm \mathrm{S}, \mathrm{n}=12)}\right.$} \\
\hline & Before exercise & After exercise & $\mathrm{P}$ & Before exercise & After exercise & $\mathrm{P}$ \\
\hline Middle men & $90.10 \pm 15.04$ & $95.33 \pm 14.02$ & 0.332 & $4.55 \pm 0.65$ & $4.19 \pm 0.61$ & 0.088 \\
Older men & $87.93 \pm 13.81$ & $107.15 \pm 9.64^{\mathbf{\Lambda}}$ & 0.001 & $4.68 \pm 0.53$ & $4.34 \pm 0.39$ & 0.118 \\
$\begin{array}{c}\text { Middle } \\
\text { women }\end{array}$ & $89.70 \pm 16.31$ & $96.35 \pm 11.83$ & 0.218 & $4.08 \pm 0.53$ & $3.77 \pm 0.49$ & 0.145 \\
$\begin{array}{c}\text { Older } \\
\text { women }\end{array}$ & $79.34 \pm 11.92$ & $95.44 \pm 11.17^{\mathbf{\Lambda}} \mathbf{\Lambda}$ & 0.003 & $4.17 \pm 0.56$ & $3.68 \pm 0.36^{\mathbf{\Lambda}}$ & 0.024 \\
\hline
\end{tabular}

Note: Compared with before exercise: $\boldsymbol{\Delta} \mathrm{P}<0.05, \boldsymbol{\Delta} \mathbf{\Delta} \mathrm{P}<0.01$.

\section{Analysis}

\subsection{The Effect of the Health Qigong New Skills on Serum SOD of Middle-Aged and Elderly Men.}

SOD is the first line of defense against free radical damage. It is an antioxidant enzyme, and its activity can reflect the strength of antioxidant capacity[4].The SOD activity decreased gradually with 
age[5].The results of this study showed that SOD activity in the serum was significantly higher than that before exercise, and there was a significant difference between the elderly men and the elderly women. The increase may be due to aerobic exercise, which increases the body's metabolism and improves circulation. Therefore, aerobic endurance exercise can improve the circulatory function and promote the synthesis of enzymes, thereby eliminating the free radicals produced in metabolism. The rest of the two groups of serum SOD activity practise after exercising is higher than before but there was no significant difference, this is the exercise intensity is small, may cause oxygen metabolism is not strong, and then to the increased amount of the increase of SOD activity or SOD synthesis stimulation is small. Prompt that old people after a period of practice, serum SOD activity increased, the body produces in the process of aerobic metabolism of oxygen free radicals can better remove, prevent free radicals oxidative damage to the body. Zhu Hanxiao[6] research also found that the activity of SOD in peripheral blood was significantly increased after 16 weeks of practice. The health Qigong $\bullet$ new skills belongs to the aerobic exercise of small and medium strength, which can improve the activity of SOD in serum and remove the $\mathrm{O}^{2-}$ damage to the body in a timely manner, so as to achieve the effect of longevity.

\subsection{The Effect of the Health Qigong New Skills on Serum MDA in Middle-Aged and Elderly People.}

The accumulation of lipids in the aging process is the result of free radical reaction. The metabolite of lipid peroxidation in organism is MDA, which has serious toxicity to cells. Therefore, the determination of MDA content in serum can reflect the degree of lipid peroxidation, which indirectly reflects the attack degree of free radical. Studies at home and abroad show that long-term aerobic exercise can reduce the serum MDA content significantly lower than the control group which can effectively inhibit the lipid peroxidation reaction, protect the body from free radical damage and delay the aging process[7]. The results of this study showed that the content of MDA in the serum was significantly lower than that in the elderly women group, and there were significant differences. The decrease may be due to the reduction of production, the degradation of LPO, the increase of transport and discharge, or the synthesis of antioxidant enzyme system. There were no significant differences in MDA content in the remaining three groups before the exercise. This may have something to do with the reasons for their work, the level of commitment to practice, and the degree of mastery of the movement's essentials. Long-term aerobic exercise can reduce the content of MDA in serum, reduce the damage degree of cell membrane, and help to delay the aging process. Liu xiaodan[8] et al. research also found that after six months of Yi Jinjing exercise, the elderly women's MDA decreased significantly. It can effectively inhibit the lipid peroxidation in the elderly and maintain the activity of free radical antioxidant enzyme in high functional level.

\section{Conclusion}

In this study, the new method practitioners were studied. The effects of serum free radical SOD and MDA metabolism were observed before and after exercise. The results show that the new achievement method as a kind of health Qigong is suitable for the activity of senile chronic aerobic exercise, which can improve the serum SOD activity and reduce MDA content in serum and reduce the degree of lipid peroxidation. Regular exercise is helpful to improve the antioxidant capacity of middle-aged and elderly people and their ability to scavenge free radicals. Prompt accurate grasp of the action essentials and a long and serious exercise in the health Qigong・new skills is good for the prevention of cardiovascular disease in the elderly and the process of slowing down the aging process.

\section{References}

[1]. Wang Ying, Chen Pang. On the Sociological Value of New Fitness Qigong Composing [J]. Wuhu Science, 2011; 8(9):103-104. 
[2]. Jin-he He, Guan-nan Liu, Zhen Chang, et al. Progress in the Study of Anti-aging Effects of Aerobic Exercise [J].Chinese Journal of Rehabilitation,2012;27(1):42-44.

[3]. Chui-kun Li, Hay-bin Dai, Ming Xu, et al. Effects of Health Qigong and New Skills on the Middle and Old Aged People's Blood Lipid[J]. Journal of Chengdu Sport University, 2013; 2(22):62-66.

[4]. Li-fen Zhang, Yu-ping Goo, Jun Zhou, et al. Effects of Exercise on Free Radical Metabolism [J]. Progress in Modern Biomedicine, 2012; 12(17):3388-3393.

[5]. Xiao-hue Ma. Traditional Chinese Medicine and Anti-decrepitude [J]. Journal of the Practical Chinese with Modern Medicine, 2005; 6(18):922-3.

[6]. Han-Xiao Zhu, Sun-young Jiao, Xue-lian Chen. The Influence of 16 Week's Wu Qinxi Exercises on Physical Function Related Indexes of Old Women [J]. Chinese Journal of Sports Medicine, 2008; 27(4):499-500.

[7]. Sheng Sheen, Kai-Xian Qi an, Shoo-in Zhang. Effects of Long Distance Running on Superoxide Dismutase and Lipid Peroxide in the Elderly [J]. Chinese Journal of Gerontology, 2004; 11(24):1024-1025.

[8]. Xiao-Dan Liu, Hong-Zhou Jin. Effect of fitness Qigong (Yi Jinjing) on blood lipid and free radical metabolism of the elder women [J]. China Journal of Traditional Chinese Medicine and Pharmacy, 2010; 25(9):1480-1482. 